\title{
A NOTE ON GALOIS GROUPS OF ALGEBRAIC CLOSURES
}

\author{
DON KRAKOWSKI
}

(Received October 1, 1972)

Communicated by J. P. O. Silberstein

A group will be called full if it is the Galois group of an algebraic closure of a field. In this paper we first investigate full Abelian groups and classify them. Then we examine full groups from the point of view of how we can operate on them and still maintain the property of being full. Of course, by the fundamental theorem of the infinite Galois theory, closed subgroups (with standard profinite topology) of full groups are full. In general, products of full groups are not full (for example, $Z_{2} \times Z_{2}$ is not full, by a theorem of Artin and Schreier (1927)); however we produce a set of groups which can always be attached as direct factors to full groups and still retain full groups. For the definition and basic properties of profinite groups we refer the reader to Cassels and Frohlich (1967). It has been shown by Leptin (1955) and independently by the author that profinite groups are Galois groups. The author (1971) has shown that if $G$ is profinite, then $G=\operatorname{Gal}(k / L)$ where $L$ may have any desired characteristic and contains all primitive $n$th roots of unity, for all $n$. We will denote by $\hat{Z}_{p}$ the pro- $p$-group which is the inverse limit of cyclic $p$-groups. (This is the group of $p$-adic integers).

We begin with a lemma which actually can be dispensed with by a slight alteration of the proof, but which we include for its independent interest.

Lemma 1. If $G$ is a profinite Abelian group, then $G$ is reduced.

Proof. Let $D$ be the maximal divisible subgroup of $G$. Then by Theorem 3 of Kaplansky (1966), $G=D \oplus R$ where $R$ is reduced. Now $D=\bigcap_{n \in Z^{+}} n G$ and since $G$ is closed each $n G$ is closed. So $D$ is closed and hence is itself a profinite group Cassels and Frohlich (1967). Therefore $D=T_{+-x} \subseteq \prod_{x \in A} T_{x}$. Now $D=n D$ for all $n \in Z^{+}$and

$$
n D \subseteq n \prod_{\alpha \in A} T_{x}=\prod_{\alpha \in A} n T_{x}
$$

So

$$
D \subseteq \sum_{n \in Z^{+}}\left(\prod_{x \in A} n T_{x}\right)=\{0\}
$$

sirce $T_{x}$ is finite for all $\propto$. So $G=R$ and hence is reduced. 
Lemma 2. If $G$ is an. Abelian profinite group, then $G \cong \prod_{\beta \in B} A_{\beta}$ where $A_{\beta}$ is finite cyclic or $A_{\beta}=\hat{Z}_{p_{\beta}}$, for all $\beta \in B$.

Proof. If $G$ is an Abelian, profinite group, then $G=G_{+\alpha}$ where we may assume $G_{\alpha}$ is Abelian. By the fundamental theorem of finite Abelian groups each $G_{\alpha}$ is the product of cyclic groups of prime power order. So $\prod_{\alpha \in A} G_{\alpha}=\prod_{i \in I} Z_{p_{i}}$. If $Z_{p_{i}}$ appears a cofinal number of times in $I$, then we have that $Z_{p_{i}}$ is a direct factor of $G$. Otherwise, we get a product of $\hat{Z}_{p}$ 's.

Lemma 3. If $G$ is a full Abelian group and if $G$ is not torsion-free, then $Z_{2}$ is a direct summand of $G$, and $G$ has no other finite groups as direct summand.

Proof. By Lemma 1, $G$ is reduced; hence if $G$ is not torsion free we use Theorem 9 of Kaplansky (1966) and obtain $G=C \oplus R$, where $C$ is a finite cyclic group. Now $C$ can be considered as a subgroup of $G$, and since all finite sets are closed in the Krull (or profinite) topology, $C$ is closed in $G$. Employing the fundamental theorem of the infinite Galois theory, since $G \simeq \operatorname{Gal}(R / K)(R$ is the algebraic closure of the field $K), C$ corresponds to a subfield $K_{c}$, and $K \subseteq K_{c} \subseteq R ; C \simeq \operatorname{Gal}\left(R / K_{c}\right)$. Now $R_{c}=R$; hence $C$ is full. By a result of Artin and Schreier (1927), the only full finite group is $Z_{2}$, and therefore $C=Z_{2}$. If $D$ were any other finite direct summand of $G$, then $D+C$ would be a finite direct summand, whence $D+C=Z_{2}$ and so $D=\{0\}$.

THForfm 1. If $G$ is a full Abelian group, then

$$
\begin{aligned}
& G \simeq Z_{2}^{\varepsilon} \times \prod_{\beta \in B} A_{\beta} \text { where } \varepsilon=0 \text { or } 1 \text { and } \\
& A_{\beta}=\dot{Z}_{p_{\beta}}, p_{\beta} \text { a prime, for each } \beta \in B \text {. }
\end{aligned}
$$

Proof. It is well known that Galois groups are profinite groups, hence our result follows from Lemmas 2 and 3 upon noting that if $G$ is torsion free then no finite group can be a direct summand.

The converse of Theorem 1 is also true as will be demonstrated later in this paper. If we let $\left\{E_{i} \mid i \in I\right\}$ be a set of Galois extensions of a field $k$, all contained in some field $K$, we will denote by $\prod_{i \in I} E_{i}$ the smallest subfield of $K$ containing all the $E_{i}$. In what follows we will need to know that if, for an ordered set $I$, we have $E_{i} \cap\left(\prod_{n \leqq i} E_{n}\right)=k$ for all $i \in I$, then

$$
\operatorname{Gal}\left(\prod_{i \in I} E_{i} / k\right) \cong \prod_{i \in I} \operatorname{Gal}\left(E_{i} / k\right) \text {. }
$$

As usual, we shall define $k((X))$ as the field of power series over $k$ in the indeterminate $X$. We shall need the following technical lemma, whose proof can be found in Serre (1968), (chapter IV, §2, Proposition 8).

Lemma 4. Let $F$ be a field of characteristic zero. Then the algebraic closure 


$$
\overline{F((X))}=F((X))\left(\left\{\theta, X^{1 / n}, \quad n=2,3, \cdots,\right\}\right),
$$

where $\theta$ is the set of algebraic elements over $F$.

THEOREM 2. Let $F$ be a field of characteristic zero and containing all $n$th roots of unity, for all $n$, and let $\mathscr{F}=F((X))\left(\left\{X^{1 / n},(n, p)=1\right\}\right)$, for $p$ a prime. Then

$$
\operatorname{Gal}(\overline{\mathscr{F}} / \mathscr{F})=\operatorname{Gal}(F / F) \times \mathcal{Z}_{p}
$$

Proof. By Lemma 4,

$$
\overline{\mathscr{F}}=F((X))\left(\left\{\theta, X^{1 / n} ; n=2,3, \cdots,\right\}\right)=\mathscr{F}\left(\left\{\theta, X^{1 / p^{*}} ; \alpha=1,2, \cdots,\right\}\right) .
$$

Let $E_{1}=\mathscr{F}(\theta)$ and $E_{2}=\mathscr{F}\left(X^{1 / p^{\alpha}} ; \alpha=1,2, \cdots\right)$. Then $E_{1}\left(E_{2}\right)=\overline{\mathscr{F}}$ and $E_{1} \cap E_{2}=\mathscr{F}$. Clearly $\operatorname{Gal}\left(E_{1} / \mathscr{F}\right) \simeq \operatorname{Gal}(F / F)$. To compute $\operatorname{Gal}\left(E_{2} / \mathscr{F}\right)$ we note that since $\mathscr{F}$ is of characteristic 0 and contains all the $n$th roots of unity,

Since

$$
\operatorname{Gal}\left(\mathscr{F}\left(X^{1 / p^{\alpha}}\right) / \mathscr{F}\right)=Z_{p^{\alpha}} \text {. }
$$

$$
E_{2}=\bigcup_{x=1}^{\infty} \mathscr{F}\left(X^{1 / p^{\alpha}}\right)
$$

we obtain $\operatorname{Gal}\left(E_{2} / \mathscr{F}\right)=\hat{Z}_{p}$. Hence

$$
\operatorname{Gal}(\overline{\mathscr{F}} / \mathscr{F}) \simeq \mathrm{Gal}\left(E_{1} / \mathscr{F}\right) \times \mathrm{Gal}\left(E_{2} / \mathscr{F}\right) \simeq \mathrm{Gal}(F / F) \times Z_{p} .
$$

Corollary. Let $F$ be as in the Theorem, and $A$ any index set. For every $\alpha \in A$ let $p_{\alpha}$ be a prime and define the tower of fields:

$$
F_{\alpha+1}=F_{\alpha}\left(\left(X_{\alpha+1}\right)\right)\left(\left\{X_{\alpha+1}^{1 / n},\left(n, p_{\alpha+1}\right)=1\right\}\right)
$$

and for limit ordinals $F_{\lambda}=\bigcup_{\alpha<\lambda} F_{\alpha}$. Let $\mathscr{F}=\bigcup_{\alpha \in A} F_{\alpha}$.

Then $\operatorname{Gal}(\overline{\mathscr{F}} / \mathscr{F}) \simeq \operatorname{Gal}(F / F)+\prod_{\alpha \in A} \hat{Z}_{p}$.

Proof. Put $E_{0}=\mathscr{F}(\theta)$, with 0 as above, and $E_{\alpha}=\mathscr{F}\left(\left\{X_{\alpha}^{1 / n}, n=2,3, \cdots\right\}\right)$. Then $E_{\alpha} \cap\left(\prod_{\alpha<\beta} E_{\beta}\right)=\mathscr{F}$ for all $\alpha, \beta \in\{0\} \cup A$, and $E_{0}\left(\prod_{\alpha \in A} E_{\alpha}\right)=\overline{\mathscr{F}}$. Now $\operatorname{Gal}\left(E_{0} / \mathscr{F}\right) \simeq \operatorname{Gal}(F, F)$, and as in the above theorem $\mathrm{Gal} E_{\alpha} / \mathscr{F}=\mathcal{Z}_{p}$. Hence since

$$
\operatorname{Gal}(\overline{\mathscr{F}} / \mathscr{F}) \simeq \operatorname{Gal}\left(E_{0}\left(\prod_{\alpha \in A} E_{\alpha}\right) / \mathscr{F}\right) \simeq \operatorname{Gal}\left(E_{0} / \mathscr{F}\right) \times \prod_{\alpha \in A} \operatorname{Gal}\left(E_{\alpha} / \mathscr{F}\right)
$$

our result follows.

Corollary. If $G$ is a full Galois group, so is $G \times \prod_{a \in A} \hat{Z}_{p q}$ for any index set $A$.

Proof. $G$ may be considered as the Galois group of a field of characteristic zero containing all primitive $n$th roots of unity. The construction above yields the result. 
RemarK. Since $Z_{2}$ and $\{e\}$ are full, this cotollary provides a proof to the converse of Theorem 1, namely, that $Z_{2}^{\ell} \times \prod_{\alpha \in A} \hat{Z}_{p_{x}}$ is full Abelian for any index set $A$.

\section{References}

E. Artin, and O. Schreier (1927), 'Eine Kennzeichnung der reell abgeschlossenen Korper', Math. Sem. Univ. Hamburg 5 225-231.

J. W. S. Cassels, and A. Frohlich (1967), Algebraic Number Theory, Chapter V by Karl Gruenberg; Profinite Groups. (Thompson Book Co. Washington D. C. 1967).

I. Kaplansky (1966), Infinite Abelian Groups, (Univ. of Mich. Press, Ann Arbor, 1966).

D. Krakowski, Profinite Groups and the Galois Groups of Fields, thesis submitted to U. C. L. A. June 1971.

H. Leptin (1955), 'Compact, totally disconnnected groups', Arch. Math. 6 371-373.

J. Serre (1968), Corps Locaux, (Hermann, Paris, 1968).

Weizmann Institute of Science

Rehovot

Israel 